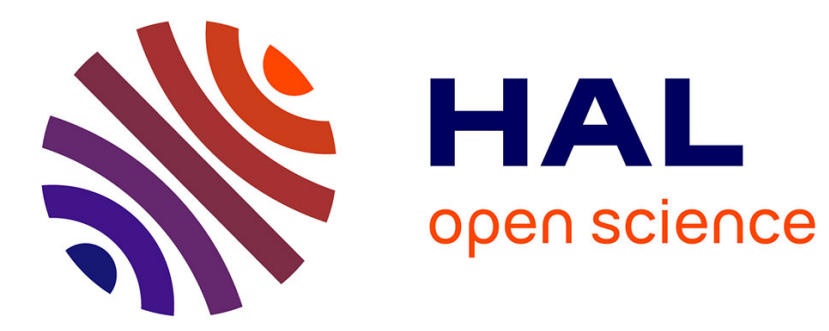

\title{
Approximate yield criteria for anisotropic metals with prolate or oblate voids
}

Vincent Monchiet, Ion-Cosmin Gruescu, Eric Charkaluk, Djimedo Kondo

\section{To cite this version:}

Vincent Monchiet, Ion-Cosmin Gruescu, Eric Charkaluk, Djimedo Kondo. Approximate yield criteria for anisotropic metals with prolate or oblate voids. Comptes Rendus Mécanique, 2006, 334, pp.431439. 10.1016/j.crme.2006.06.001 . hal-00090592

\section{HAL Id: hal-00090592 https://hal.science/hal-00090592}

Submitted on 27 Jul 2021

HAL is a multi-disciplinary open access archive for the deposit and dissemination of scientific research documents, whether they are published or not. The documents may come from teaching and research institutions in France or abroad, or from public or private research centers.
L'archive ouverte pluridisciplinaire HAL, est destinée au dépôt et à la diffusion de documents scientifiques de niveau recherche, publiés ou non, émanant des établissements d'enseignement et de recherche français ou étrangers, des laboratoires publics ou privés. 


\title{
Approximate yield criteria for anisotropic metals with prolate or oblate voids
}

\author{
Vincent Monchiet, Cosmin Gruescu, Eric Charkaluk, Djimedo Kondo* \\ Laboratoire de mécanique de Lille-UMR CNRS 8107, université de sciences et technologies Lille, \\ cité scientifique, boulevard Paul-Langevin, 59655 Villeneuve d'Ascq cedex, France
}

\begin{abstract}
Following the study of Gologanu et al. (1997) which has extended the well-known approach of Gurson (1975), we propose approximate yield criteria for anisotropic plastic voided metals containing non spherical cavities. The plastic anisotropy of the matrix is described by means of Hill's quadratic criterion. The procedure to establish the closed form expression of approximate macroscopic criteria, in which void shape and plastic anisotropic effects are included, is detailed. The new criteria allow us to recover existing results in the cases of spherical and cylindrical voids in an Hill type plastic matrix. Moreover, they agree with previous criteria for non spherical voids in an isotropic plastic matrix. Finally, for validation purposes, we provide, in the general case of non spherical cavities in the anisotropic matrix, a comparison with the numerical exact two field criteria.
\end{abstract}

\section{Résumé}

Critères macroscopiques pour des métaux plastiques anisotropes contenant des cavités non sphériques. En se basant sur les travaux de Gologanu et al. (1997) qui étendent l'approche bien connue de Gurson, on propose des critères macroscopiques pour des métaux plastiques anisotropes contenant des cavités non sphériques. L'anisotropie plastique de la matrice est décrite à l'aide du critère quadratique de Hill. On détaille la procédure pour établir les critères approchés incluant la forme des cavités ainsi que les effets d'anisotropie plastique. Les nouveaux critères analytiques obtenus permettent de retrouver les résultats existants dans les cas de cavités sphériques ou cylindriques dans une matrice de type Hill. De plus, ils concordent avec ceux qui sont disponibles pour des cavités non sphériques dans un milieu plastique isotrope. Enfin, dans un but de validation, on fournit, dans le cas général de cavités non sphériques dans la matrice anisotrope, une comparaison avec les expressions des critères issues des solutions numériques exactes du problème à deux champs.

Keywords: Computational solid mechanics; Ductile porous metals; Anisotropy; Hill criterion; Prolate and oblate voids

Mots-clés : Mécanique des solides numérique; Métaux ductiles poreux ; Anisotropie ; Critère de Hill ; Cavités allongées et aplaties

\footnotetext{
* Corresponding author.

E-mail addresses: vincent.monchiet@ed.univ-lille1.fr (V. Monchiet), ic.gruescu@univ-lille1.fr (C. Gruescu), eric.charkaluk@univ-lille1.fr (E. Charkaluk), kondo@univ-lille1.fr (D. Kondo).
} 


\section{Introduction}

Void nucleation, growth and coalescence are commonly recognized as the basic micromechanisms of the ductile fracture of metals. Since the pioneering work of Gurson [1] for a hollow sphere in a von Mises perfect plastic matrix, the modelling of voids growth has been the subject of several works performed in the context of ductile damage mechanics. Gologanu et al. [2,3] (see also [4]), Garajeu et al. [5] provide various extensions of the Gurson model by incorporating void shape effects; either prolate and oblate voids are considered in these studies. Other recent extensions of the Gurson model concern the consideration of plastic anisotropy. For instance, [6-8] deal with void growth in a metal matrix, obeying the Hill quadratic criterion [9]. Unfortunately, all these studies are limited to the case of spherical and cylindrical voids. The purpose of the present Note is to provide a closed form approximated expression of the macroscopic yield function of anisotropic metals containing non spherical voids. Practical applications of the new criteria can be found in the domain of metal forming (see [10]).

\section{Principle of the estimate of the macroscopic yield criterion}

\subsection{Statement of the problem}

Following Gologanu et al. [4], consider a spheroidal (axisymmetric) prolate or oblate cavity with semi-axes $a_{1}$ (along $\underline{e}_{3}$ ), and $b_{1}$ (along $\underline{e}_{1}$ and $\underline{e}_{2}$ ) embedded in a cell which has the shape of a confocal spheroid with the semiaxes $a_{2}$ (along $\underline{e}_{3}$ ), and $b_{2}$ (along $\underline{e}_{1}$ and $\underline{e}_{2}$ ). $a_{1}>b_{1}$ corresponds to a prolate cavity while $b_{1}>a_{1}$ is associated to an oblate one. Let us denote $c$ the focal distance and $e_{1}$ the eccentricity defined by: $c=\sqrt{a_{1}^{2}-b_{1}^{2}}, e_{1}=c / a_{1}$ for a prolate and $c=\sqrt{b_{1}^{2}-a_{1}^{2}}, e_{1}=c / b_{1}$ for an oblate cavity (see Fig. 1).

For this class of geometry, it is convenient to introduce the system of spheroidal coordinates characterized by $\lambda, \beta, \varphi$, defined, in the cylindrical frame (coordinates $\rho, \varphi, z$ ), by: $\rho=c \sinh \lambda \sin \beta$ and $z=c \cosh \lambda \cos \beta$ for a prolate; in the case of an oblate, one has $\rho=c \cosh \lambda \sin \beta$ and $z=c \sinh \lambda \cos \beta$.

The iso- $\lambda$ surface defines confocal spheroids, with semi axes $a=c \cosh (\lambda), b=c \sinh (\lambda)$ and eccentricity $e=c / a$, for a prolate. An oblate spheroid is associated to semi axes $a=c \sinh (\lambda), b=c \cosh (\lambda)$ and eccentricity $c / b$. The unit vectors of the new base are:

$$
\underline{e}_{\lambda}=\frac{1}{L_{\lambda}}\left\{a \sin (\beta) \underline{e}_{\rho}+b \cos (\beta) \underline{e}_{3}\right\} ; \quad \underline{e}_{\beta}=\frac{1}{L_{\lambda}}\left\{b \cos (\beta) \underline{e}_{\rho}-a \sin (\beta) \underline{e}_{3}\right\} ; \quad \underline{e}_{\varphi}=\underline{e}_{\varphi}
$$

with: $L_{\lambda}=\sqrt{a^{2} \sin ^{2}(\beta)+b^{2} \cos ^{2}(\beta)}, \varphi \in[0,2 \pi], \beta \in[0, \pi]$ and $\underline{e}_{\rho}=\cos (\beta) \underline{e}_{1}+\sin (\beta) \underline{e}_{2}$. The porosity is defined by: $f=a_{1} b_{1}^{2} /\left(a_{2} b_{2}^{2}\right)$.

The velocity field, $\underline{v}$, in the matrix is classically decomposed in a uniform strain rate, $\boldsymbol{A} . \underline{x}$, and an inhomogeneous field $\underline{v}^{E}: \underline{v}=A \cdot \underline{x}+\bar{B} \underline{v}^{E}$. We adopt the trial velocity field used by [2] and [3] for nonspherical cavities analysis in
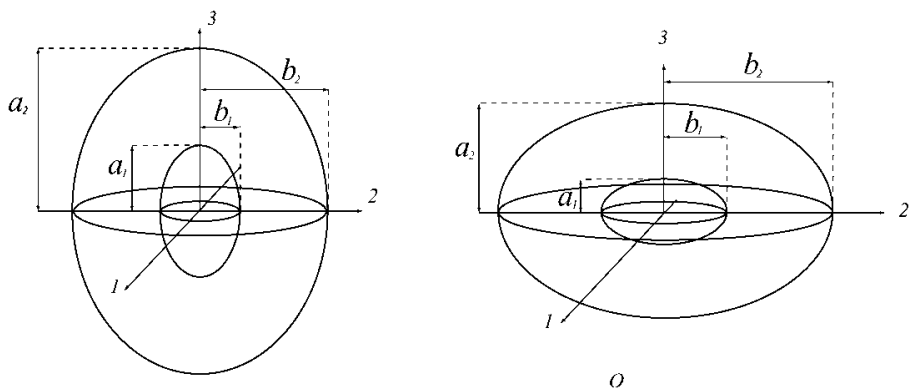

Fig. 1. The geometry of the cell considered: (left) prolate cavity; (right) oblate cavity.

Fig. 1. Géométrie de la cellule considérée : (à gauche) cavité allongée; (à droite) cavité aplatie. 
a von-Mises matrix. Even though the accuracy expected from this choice is questionable in the anisotropic case, ${ }^{1}$ the estimate of the macroscopic plastic dissipation being greater than the exact one, it is expected that the approximate yield surface will be external to the exact one and will probably need to be improved in further work.

By considering the uniform strain rate condition on the cell boundary, $\underline{v}\left(\lambda=\lambda_{2}\right)=\boldsymbol{D} \cdot \underline{x}(\boldsymbol{D}$ being the macroscopic strain rate), $\boldsymbol{A}$ and $B$ can be identified. Indeed, denoting $D_{h} \mathbf{1}$ the hydrostatic part of $\boldsymbol{D}$ :

$$
\boldsymbol{A}=\boldsymbol{D}-D_{h} \boldsymbol{X} ; \quad B=\frac{a_{2} b_{2}^{2}}{c^{3}} D_{h} \quad \text { with: } \boldsymbol{X}=\frac{3}{2}\left(1-\alpha_{2}\right)\left(\underline{e}_{1} \otimes \underline{e}_{1}+\underline{e}_{2} \otimes \underline{e}_{2}\right)+3 \alpha_{2} \underline{e}_{3} \otimes \underline{e}_{3}
$$

$\alpha_{2}=\alpha\left(e_{2}\right) . \alpha(e)$, the function of eccentricity $e$, is defined by:

$$
\alpha(e)= \begin{cases}\frac{1-e^{2}}{e^{3}}(\operatorname{arctanh}(e)-e) & \text { (prolate) } \\ \frac{e-\arcsin (e) \sqrt{1-e^{2}}}{e^{3}} & \text { (oblate) }\end{cases}
$$

The strain rate field is defined by $\boldsymbol{d}=\boldsymbol{A}+B \boldsymbol{d}^{E}$, for which $\boldsymbol{d}^{E}$ is obtained from the inhomogeneous velocity field $\underline{v}^{E}$ in the spheroidal coordinates. Let us recall that we adopt the velocity fields proposed by [2] and [3], instead of those provided in [4]. It follows that:

$$
\begin{aligned}
\boldsymbol{d}^{E}= & \frac{3 c^{3}(1-\alpha)}{2 a b^{2}}\left(1-3 \underline{e}_{\lambda} \otimes \underline{e}_{\lambda}\right)+\frac{3 a c^{3}(1-3 \alpha)}{2 b^{2} L_{\lambda}^{2}} \sin ^{2}(\beta)\left(\underline{e}_{\lambda} \otimes \underline{e}_{\lambda}-\underline{e}_{\beta} \otimes \underline{e}_{\beta}\right) \\
& +\frac{3 c^{3}(1-3 \alpha)}{2 b L_{\lambda}^{2}} \sin (2 \beta) \underline{e}_{\lambda} \stackrel{s}{\otimes} \underline{e}_{\beta}
\end{aligned}
$$

\subsection{Principle of the determination of the macroscopic criteria}

We consider now that the plastic matrix obeys the Hill quadratic criterion which can be expressed:

$$
F\left(\sigma_{11}-\sigma_{22}\right)^{2}+G\left(\sigma_{22}-\sigma_{33}\right)^{2}+H\left(\sigma_{33}-\sigma_{11}\right)^{2}+2 L \sigma_{23}^{2}+2 M \sigma_{13}^{2}+2 N \sigma_{12}^{2}-\frac{2}{3} \sigma_{0}^{2} \leqslant 0
$$

in which $F, G, \ldots, N$ are material constants and $\sigma_{0}$ the yield stress of the matrix. $\sigma$ denotes the microscopic stress field. This expression, written in the frame of material anisotropy, can be translated as:

$$
\mathcal{F}(\sigma)=\frac{3}{2} \sigma: \mathbb{M}: \sigma-\sigma_{0}^{2} \leqslant 0
$$

$\mathbb{M}$ is a general ${ }^{2}$ fourth order anisotropic tensor whose components are expressed in the frame of the spheroid $\left(\underline{e}_{1}, \underline{e}_{2}, \underline{e}_{3}\right)$. When the voids orientation and the direction of plastic anisotropy coincide, $\mathbb{M}$ is orthotropic in nature. In any case, the plastic incompressibility condition in the matrix reads $M_{i i k l}=0$. Let us recall now the definition of the dissipation:

$$
\Pi(\boldsymbol{D})=\frac{\sigma_{0}}{|\Omega|} \int_{\Omega} d_{\mathrm{eq}} \mathrm{d} V
$$

in which $\Omega$ denotes the considered domain whose volume is $|\Omega|=4 \pi a_{2} b_{2}^{2} / 3 ; d_{\mathrm{eq}}$ is the microscopic equivalent plastic strain rate which is written:

$$
d_{\mathrm{eq}}^{2}=\frac{2}{3} \boldsymbol{d}: \mathbb{H}: \boldsymbol{d}=A_{\mathrm{eq}}^{2}+\frac{4}{3} \boldsymbol{A}: \mathbb{H}: \boldsymbol{d}^{E}+d_{\mathrm{eq}}^{E^{2}}
$$

\footnotetext{
1 Indeed, the isotropic expansion field is no longer the exact field under hydrostatic loading. However, note that, by adopting this trial velocity fields, we follow the same kind of consideration as in [6] for spherical voids and as in [7] for cylindrical ones in the context of plastic anisotropy.

2 Such generality allows us to consider arbitrarily orientated voids with respect to the plastic anisotropy direction. Note also that the (isotropic) von Mises criterion of the matrix is obtained from (6) by putting $\mathbb{M}=\mathbb{K}=\mathbb{I}-\mathbb{J}$ where $\mathbb{I}$ is the fourth order symmetric identity tensor and $\mathbb{J}=\frac{1}{3} \mathbf{1} \otimes \mathbf{1}$.
} 
$\mathbb{H}$ is the fourth order tensor defined by $\mathbb{H}: \mathbb{M}=\mathbb{K}$; it also satisfies the matrix incompressibility condition, $H_{i i k l}=0$. Expressions of $H_{i j k l}$ as function of the components of $\mathbb{M}$ are given in Appendix A. The yield surface related to the macroscopic dissipation is classically deduced from the two following relations:

$$
3 \Sigma_{h}=\frac{\partial \Pi}{\partial D_{h}}=\frac{\partial \Pi}{\partial \boldsymbol{A}}:(\mathbf{1}-\boldsymbol{X})+\frac{\partial \Pi}{\partial B} \frac{a_{2} b_{2}^{2}}{c^{3}} \quad \text { and } \quad \overline{\boldsymbol{\Sigma}}=\frac{\partial \Pi}{\partial \overline{\boldsymbol{D}}}=\frac{\partial \Pi}{\partial \boldsymbol{A}}: \frac{\partial \boldsymbol{A}}{\partial \overline{\boldsymbol{D}}}+\frac{\partial \Pi}{\partial B} \frac{\partial B}{\partial \overline{\boldsymbol{D}}}=\frac{\partial \Pi}{\partial \boldsymbol{A}}
$$

which, by combination, leads to:

$$
3 \Sigma_{h}^{*}=\boldsymbol{\Sigma}: \boldsymbol{X}=\frac{\partial \Pi}{\partial B} \frac{a_{2} b_{2}^{2}}{c^{3}} ; \quad \text { and } \quad \overline{\boldsymbol{\Sigma}}=\frac{\partial \Pi}{\partial \boldsymbol{A}}
$$

$\overline{\boldsymbol{D}}$ and $\overline{\boldsymbol{\Sigma}}$ are the deviatoric part of the macroscopic strain rate and the macroscopic stress respectively. Due to the difficulty of integrating the microscopic dissipation on $\Omega$ (Eq. (7)), the purpose of the next section is to provide some approximate expressions of the macroscopic dissipation and then of the yield criteria, either for prolate and oblate voids in anisotropic plastic matrix.

\section{Macroscopic yield criteria for an anisotropic porous material}

\subsection{Approximate expression of the macroscopic dissipation}

In order to establish an approximate expression of the macroscopic criteria by using (10), an analytical expression of the $\Pi(\boldsymbol{D})$ is due. In the spheroidal frame, one has:

$$
\Pi(\boldsymbol{D})=\frac{1}{|\Omega|} \int_{\Omega} d_{\mathrm{eq}} \mathrm{d} V=\frac{3 c^{3}}{4 \pi a_{2} b_{2}^{2}} \int_{\lambda=\lambda_{1}}^{\lambda=\lambda_{2}} \int_{\beta=0}^{\beta=\pi} \int_{\varphi=0}^{\varphi=2 \pi} d_{\mathrm{eq}} b L_{\lambda}^{2} \sin \beta \mathrm{d} \lambda \mathrm{d} \beta \mathrm{d} \varphi
$$

The first step of the approximation consists of replacing $d_{\mathrm{eq}}$ by a mean value along each confocal spheroid to the cavity. It follows that (11) can be put in the form:

$$
\Pi(\boldsymbol{D})=\frac{c^{3}}{a_{2} b_{2}^{2}} \int_{\lambda=\lambda_{1}}^{\lambda=\lambda_{2}}\left\{\left\langle d_{\mathrm{eq}}^{2}\right\rangle_{\mathcal{E}}\right\}^{1 / 2} b\left(2 a^{2}+b^{2}\right) \mathrm{d} \lambda
$$

The proposal made for (12) consists in replacing $d_{\mathrm{eq}}^{2}$ by the following mean value on each confocal spheroid:

$$
\left\langle d_{\mathrm{eq}}^{2}\right\rangle_{\mathcal{E}}=\frac{3}{4 \pi\left(2 a^{2}+b^{2}\right)} \int_{\beta=0}^{\beta=\pi} \int_{\varphi=0}^{\varphi=2 \pi} \mathrm{d}_{\mathrm{eq}}^{2} L_{\lambda}^{2} \sin (\beta) \mathrm{d} \beta \mathrm{d} \varphi
$$

This is motivated by the fact that, contrarily to the isotropic case, $d_{\mathrm{eq}}$ is function of $\varphi$ in the context of plastic anisotropy. Using (8), $\left\langle d_{\mathrm{eq}}^{2}\right\rangle_{\mathcal{E}}$ reads:

$$
\left\langle d_{\mathrm{eq}}^{2}\right\rangle_{\mathcal{E}}=A_{\mathrm{eq}}^{2}+\frac{4}{3} \boldsymbol{A}: \mathbb{H}:\left\langle\boldsymbol{d}^{E}\right\rangle_{\mathcal{E}}+\left\langle d_{\mathrm{eq}}^{E^{2}}\right\rangle_{\mathcal{E}}
$$

with

$$
\left\langle\boldsymbol{d}^{E}\right\rangle_{\mathcal{E}}=\frac{3}{4 \pi\left(2 a^{2}+b^{2}\right)} \int_{\beta=0}^{\beta=\pi} \int_{\varphi=0}^{\varphi=2 \pi} \boldsymbol{d}^{E} L_{\lambda}^{2} \sin (\beta) \mathrm{d} \beta \mathrm{d} \varphi=\frac{3 Q(e)}{2} \boldsymbol{Q}
$$

where $Q$ is a second order tensor and $Q(e)$ is a function of eccentricity $e$. With $\alpha=\alpha(e)$ given by (3):

$$
Q=-\frac{1}{2}\left(\underline{e}_{1} \otimes \underline{e}_{1}+\underline{e}_{2} \otimes \underline{e}_{2}\right)+\underline{e}_{3} \otimes \underline{e}_{3} ; \quad Q(e)= \begin{cases}2 \frac{3 \alpha-1+e^{2}(1-\alpha)}{\left(1-e^{2}\right)\left(3-e^{2}\right)} e^{3} & \text { (prolate) } \\ 2 \frac{3 \alpha-1-2 e^{2} \alpha}{\sqrt{1-e^{2}}\left(3-2 e^{2}\right)} e^{3} & \text { (oblate) }\end{cases}
$$


The mean value of $d_{\mathrm{eq}}^{2}$ on each confocal spheroid reads:

$$
\left\langle d_{\mathrm{eq}}^{E^{2}}\right\rangle_{\mathcal{E}}=P(e)
$$

For prolate cavities, one has:

$$
\begin{aligned}
P(e)= & \frac{3}{2} \frac{e^{4}}{\left(1-e^{2}\right)^{2}\left(3-e^{2}\right)}\left\{\left(3 e^{2} \alpha(1-\alpha)-1+3 \alpha\right) h_{1}\right. \\
& \left.+9\left(1-e^{2}+\alpha e^{2}\right)\left(e^{2}(1-\alpha)-1+3 \alpha\right) h_{2}+4(1-3 \alpha)\left(1-e^{2}\right) h_{3}\right\}
\end{aligned}
$$

and for oblate cavities:

$$
P(e)=\frac{3}{2} \frac{e^{4}}{3-2 e^{2}}\left\{\left(1-3 \alpha+2 e^{2}\right) h_{1}+9 \frac{1-\alpha e^{2}}{1-e^{2}}\left(1-3 \alpha+2 \alpha e^{2}\right) h_{2}-4(1-3 \alpha) h_{3}\right\}
$$

where parameters $h_{i}$ are related to the anisotropy coefficients (using Voigt's notations) by:

$$
h_{1}=H_{11}+H_{22}+H_{66}-\frac{1}{2} H_{33}, h_{2}=H_{33}, h_{3}=H_{44}+H_{55}
$$

To summarize, $\left\langle d_{\mathrm{eq}}^{2}\right\rangle_{\mathcal{E}}$, given by (14), takes the form:

$$
\left\langle d_{\mathrm{eq}}^{2}\right\rangle_{\mathcal{E}}=A_{\mathrm{eq}}^{2}+2 Q(e) B \boldsymbol{A}: \mathbb{H}: \boldsymbol{Q}+P(e) B^{2}
$$

The determination of the macroscopic dissipation requires now the integration of (12) with (21) over the variable $\lambda \in\left[\lambda_{1}, \lambda_{2}\right]$. Once again, we need to approximate the result. Let us introduce the following notations: $\mu_{s}=\sqrt{\frac{2 h_{1}+3 h_{2}+2 h_{3}}{10}} ; \mu_{c}=\sqrt{\frac{3 h_{1}}{8}}$ and $\mu_{p}=\sqrt{3 h_{3}+\frac{27 \pi^{2}}{32} h_{2}}$.

In the case of prolate cavities, it is convenient to apply the following change of variable $x=c^{3} /\left(a b^{2}\right)$. It is then easy to check that $P(e) \simeq 4 \mu_{s}^{2} x^{2}$ for $x \rightarrow 0$ and $P(e) \simeq 4 \mu_{c}^{2} x^{2}$ for $x \rightarrow+\infty$. It appears that $P(e)$ can be considered approximately 'proportional' to $x^{2}$. For oblate cavities, it is easy to check that $P(e) \simeq 4 \mu_{s}^{2} x^{2}$ for $x \rightarrow 0$ and $P(e) \rightarrow$ $4 \mu_{p}^{2}$ for $x \rightarrow+\infty$. The following change of variable is then introduced: $y=\frac{\mu_{p} x}{x+\mu_{p}} . P(e)$ is then approximately 'proportional' to $y^{2}$.

As a unified notation, let us introduce $u$ such that $u=x$ for prolate cavities and $u=y$ for oblate cavities. The most important approximation is made now, in order to replace (12) by an integral of the type $\int \sqrt{F^{2}+G^{2} u^{2}} \mathrm{~d} u / u^{2}$. Following [4], $P(e)$ and $Q(e)$ are respectively replaced by $p^{2}(e) u^{2}$ and $q(e) u^{2}$. Since $p(e)$ exhibits low variation according to $e$, it is replaced by a mean value which we have to be evaluate later. According to [4], $q(e)$ is also replaced by a constant. Although this approximation is not completely justified, $q(e) \rightarrow+\infty$ when $e \rightarrow 0$, it was observed in the isotropic case that it gives particularly good results for the approximate criteria. Using the above approximations, $d_{\mathrm{eq}}^{2}$ becomes:

$$
d_{\mathrm{eq}}^{2}=A_{\mathrm{eq}}^{2}-\frac{q^{2} u^{2}}{p^{2}}(\boldsymbol{A}: \mathbb{H}: \boldsymbol{Q})^{2}+\left\{p B+\frac{q}{p} \boldsymbol{A}: \mathbb{H}: \boldsymbol{Q}\right\}^{2} u^{2}
$$

Since the effect of the term $\frac{q^{2} u^{2}}{p^{2}}$ is low, it can also be replaced by a constant, denoted by convenience $\frac{2 r}{3 p^{2}}$. The macroscopic dissipation is then expressed as:

$$
\Pi(\boldsymbol{D})=\sigma_{0} x_{2} \int_{u_{1}}^{u_{2}}\left\{\tilde{A}^{2}+\widetilde{B}^{2} u^{2}\right\}^{1 / 2} \frac{\mathrm{d} u}{u^{2}}=\sigma_{0} x_{2}\left[\widetilde{B} \operatorname{arcsinh}\left\{\frac{u \widetilde{B}}{\tilde{A}}\right\}-\frac{\sqrt{\tilde{A}^{2}+u^{2} \widetilde{B}^{2}}}{u}\right]_{u_{1}}^{u_{2}}
$$

for which is used the following change of variable: $\tilde{A}^{2}=A_{\text {eq }}^{2}-\frac{2 r}{3 p^{2}}(\boldsymbol{A}: \mathbb{H}: \boldsymbol{Q})^{2}$ and $\widetilde{B}=p B+\frac{q}{p}(\boldsymbol{A}: \mathbb{H}: \boldsymbol{Q})$. It is recalled that $u=x$ for a prolate cavity and $u=y$ for an oblate one. 


\subsection{Determination of the yield function}

We have to determine now the macroscopic yield surface by reporting (23) in (10), using the following change of variables $(A, B \Rightarrow \tilde{A}, \widetilde{B})$ :

$$
3 \Sigma_{h}^{*}=\frac{p}{x_{2}} \frac{\partial \Pi}{\partial \widetilde{B}} ; \quad \overline{\boldsymbol{\Sigma}}-\frac{3 q x_{2}}{p^{2}} \Sigma_{h}^{*} \mathbb{H}: \boldsymbol{Q}=\frac{\partial \Pi}{\partial \tilde{A}} \frac{\partial \tilde{A}}{\partial \boldsymbol{A}}
$$

In order to ease the expression of the yield surface, it is useful to put $\tilde{A}^{2}$ in the form $\tilde{A}^{2}=\frac{2}{3} \boldsymbol{A}: \mathbb{H}^{*}: \boldsymbol{A}$, with $\mathbb{H}^{*}=$ $\mathbb{H}-\frac{r}{p^{2}}(\mathbb{H}: \boldsymbol{Q}) \otimes(\mathbb{H}: \boldsymbol{Q})$. Let us denote by $\Sigma_{\text {eq }}^{*}$, an equivalent stress defined by:

$$
\begin{aligned}
& \left(\Sigma_{\text {eq }}^{*}\right)^{2}=\frac{3}{2}\left\{\overline{\boldsymbol{\Sigma}}-\frac{3 q x_{2}}{p^{2}} \Sigma_{h}^{*} \mathbb{H}: \boldsymbol{Q}\right\}: \mathbb{M}^{*}:\left\{\overline{\boldsymbol{\Sigma}}-\frac{3 q x_{2}}{p^{2}} \Sigma_{h}^{*} \mathbb{H}: \boldsymbol{Q}\right\}=\left(\frac{\partial \Pi}{\partial \tilde{A}}\right)^{2} \\
& \text { with: } \mathbb{M}^{*}=\mathbb{M}+\frac{r}{p^{2}-r \boldsymbol{Q}: \mathbb{H}: \boldsymbol{Q}} \boldsymbol{Q} \otimes \boldsymbol{Q}
\end{aligned}
$$

such that $\mathbb{M}^{*}: \mathbb{H}^{*}=\mathbb{K}$. For prolate cavities, $u_{2}=x_{2}$ and $u_{1}=x_{1}=x_{2} / f$. For oblate cavities, let us introduce $g$ defined by $g=x_{2} / \mu_{p}$; this implies $x_{1}=\mu_{p} g / f, y_{2}=\mu_{p} g /(g+1)$ and $y_{1}=\mu_{p} g /(g+f)$.

The yield surface, deduced from (24) for prolate and oblate voids, takes the general form:

$$
\mathcal{F}^{\text {macro }}=\left(\frac{\Sigma_{\text {eq }}^{*}}{\sigma_{0}}\right)^{2}+2(1+g)(f+g) \cosh \left\{\frac{3 \Sigma_{h}^{*}}{p \sigma_{0}}\right\}-(1+g)^{2}-(f+g)^{2}
$$

with $g$ conventionally taken as zero $(g=0)$ in the case of prolate voids. In order to make explicit (26) which is the most important result of the study, a more detailed expression of $\Sigma_{\mathrm{eq}}^{*}$ is required, using (25):

$$
\Sigma_{\text {eq }}^{* 2}=\Sigma_{\text {eq }}^{2}+k_{1}\left(\Sigma_{h}^{*}\right)^{2}+k_{2} \Sigma: Q^{2}-2 k_{3} \Sigma_{h}^{*} \Sigma: Q ; \quad \Sigma_{h}^{*}=\frac{1}{3} \Sigma: X
$$

where $\boldsymbol{X}$ is given by (2). $k_{1}, k_{2}$ and $k_{3}$ depend on $p, q, r$ and $\boldsymbol{Q}: \mathbb{H}: \boldsymbol{Q}=9 h_{2} / 4$ as:

$$
k_{1}=\frac{243}{2 p^{2}} \frac{\kappa^{2} h_{2}}{4 p^{2}-9 r h_{2}} ; \quad k_{2}=\frac{6 r}{4 p^{2}-9 r h_{2}} ; \quad k_{3}=\frac{18 \kappa}{4 p^{2}-9 r h_{2}} ;
$$

with $\kappa=q x_{2}$. The macroscopic yield criterion is completely defined by (26), (27) and (28) in which $p, r$ and $\kappa$ have to be determined; this is the subject of the following two subsections.

\subsection{Calculation of the coefficient $p$}

We now come to the determination of the formulae which will give the coefficient $p$. An efficient method used by [4] in the isotropic case consists in considering the exact value in the particular case where $A_{\text {eq }}=0$. For the plastic anisotropic matrix case, $p(e)$ is also function of $h_{1}, h_{2}$ and $h_{3}$ and the above procedure cannot be used. We propose therefore to identify $p$ as follows:

$$
p^{2}=\frac{1}{u_{2}-u_{1}} \int_{u_{1}}^{u_{2}} p^{2}(e) \mathrm{d} u
$$

With the notation $\alpha_{1}=\alpha\left(e_{1}\right)$ and $\alpha_{2}=\alpha\left(e_{2}\right)$, this leads, for prolate voids (using $u=x$ ), to:

$$
\begin{aligned}
p^{2}= & \frac{3}{4} \frac{1}{1-f}\left\{\left\{\frac{1-3 \alpha_{1}}{e_{1}^{2}}-f \frac{1-3 \alpha_{2}}{e_{2}^{2}}\right\}\left(h_{1}+9 h_{2}-4 h_{3}\right)\right. \\
& \left.+18\left\{\alpha_{1}\left(1-\alpha_{1}\right)-f \alpha_{2}\left(1-\alpha_{2}\right)\right\} h_{2}+\left\{1-\alpha_{1}-f\left(1-\alpha_{2}\right)\right\}\left(h_{1}-9 h_{2}+4 h_{3}\right)\right\}
\end{aligned}
$$

and for oblate voids (using $u=y$ ): 


$$
\begin{aligned}
p^{2}= & \frac{3}{4} \frac{(1+g)(f+g)}{f(1-f)}\left\{\left\{f \frac{1-3 \alpha_{2}}{e_{2}^{2}}-\frac{1-3 \alpha_{1}}{e_{1}^{2}}\right\}\left(h_{1}+9 h_{2}-4 h_{3}\right)\right. \\
& \left.+2(1-f) h_{1}+18\left\{f \alpha_{2}^{2}-\alpha_{1}^{2}\right\} h_{2}+4\left(f \alpha_{2}-\alpha_{1}\right)\left(h_{1}-2 h_{3}\right)\right\}
\end{aligned}
$$

\subsection{Calculation of the coefficient $\kappa$ and $r$}

The procedure used consists of identifying $\kappa$ and $r$ with the two fields exact solution in the particular case $B \rightarrow 0$. The identification method, given in Appendix B, leads to: ${ }^{3}$

$$
\kappa=\frac{2(1+g)(f+g)\left(\alpha_{2}-\alpha_{1}\right)}{(1-f)} ; \quad r=\frac{6(1+g)(f+g)\left(\alpha_{2}-\alpha_{1}\right)^{2}}{(1-f)^{2}}
$$

for which, we recall that $g=0$ for prolate voids. It is interesting to notice that in the expression of $\kappa=q x_{2}, q$ could be also deduced from the same procedure used for the determination of $p$.

\section{Validation}

A first step of validation of the results is performed by considering special cases. Indeed, for the hollow sphere which is the particular case of a prolate or an oblate corresponding to $e_{1}=e_{2}=0$, this implies that $\kappa=r=g=0$ and then $\Sigma_{\text {eq }}^{*}=\Sigma_{\text {eq }}$ and $\Sigma_{h}^{*}=\Sigma_{h}$ and $p^{2}=\frac{2}{5}\left(2 h_{1}+3 h_{2}+2 h_{3}\right)=\frac{4}{5}\left(H_{11}+H_{22}+H_{33}+H_{44}+H_{55}+H_{66}\right)$. This is exactly the result already established by [6].

The case of cylindrical voids corresponds to the following limiting case of prolate voids: $e_{1}=e_{2}=1$; then $\kappa=$ $r=0, \Sigma_{\text {eq }}^{*}=\Sigma_{\text {eq }}, \Sigma_{h}^{*}=\left(\Sigma_{x x}+\Sigma_{y y}\right) / 2$ and $p^{2}=\frac{3 h_{1}}{2}=\frac{3}{2}\left(H_{11}+H_{22}-\frac{1}{2} H_{33}+H_{66}\right)$.

We also mention that for an isotropic matrix $\left(h_{1}=h_{3}=2\right.$ and $\left.h_{2}=2 / 3\right), p=2$ for spherical voids and $p=\sqrt{3}$ for cylindrical voids which is in agreement with Gurson's results. We now propose a comparison between the approximate
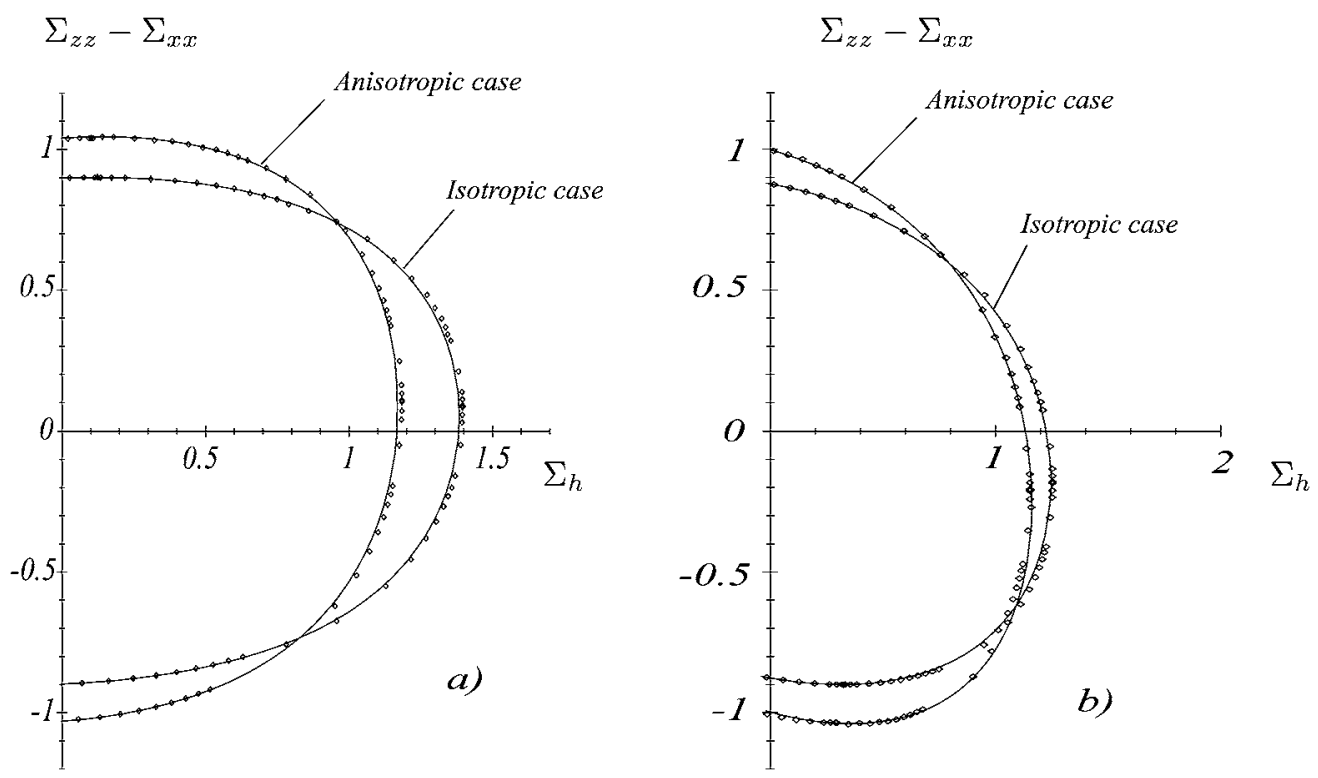

Fig. 2. Comparison between isotropic and anisotropic yield locus for: (a) prolate cavity, $a_{1} / b_{1}=5, f=0.1$, (b) oblate cavity $b_{1} / a_{1}=5, f=0.1$. The results (Eq. (26)) are compared to the two field exact ones (full line).

Fig. 2. Comparaison des seuils pour le cas isotrope et pour le cas anisotrope : (a) cavité allongée, $a 1 / b 1=5, f=0,1$, (b) cavité aplatie $b 1 / a 1=5$, $f=0,1$. Les résultats (Éq. (26)) sont comparés aux résultats exacts de la méthode à deux champs (trait plein).

\footnotetext{
3 The solutions are very closed to those obtained in [4], and can be deduced by developing hyperbolic terms which appear in their expressions, to the second order.
} 
two fields criteria (26) (full line) and the numerical exact two field criteria (discrete points) on Fig 2. The yield locus for prolate $\left(a_{1} / b_{1}=5\right)$ and oblate $\left(b_{1} / a_{1}=5\right)$ cavities are represented on Figs. 2(a) and 2(b) respectively, for either the particular case of the isotropic von Mises matrix and for the anisotropic Hill matrix. Anisotropy coefficients used for the numerical applications are: $M_{11}=0.733, M_{22}=0.57, M_{33}=0.499, M_{44}=3.669, M_{55}=1.141, M_{66}=2.2$. The data considered come from [6]. The results clearly show the effects of the plastic anisotropy of the matrix. However, a complete validation of the new results (Eq. (26)) requires a larger number of velocity fields and probably finite elements computations. This can be the purpose of a forthcoming study.

\section{Appendix A. Expression of components of $\mathbb{H}$}

For completeness, let us first indicate the relations between the components of $\mathbb{H}$ and the ones of $\mathbb{M}$. Let us first note that in the local frame of the ellipsoidal void $\left(\underline{e}_{1}, \underline{e}_{2}, \underline{e}_{3}\right)$ the components of the tensors are obtained from usual transformation rules: $H_{i j k l}=R_{i m} R_{j n} R_{k p} R_{l q} H_{m n p q}^{0}$ and $M_{i j k l}=R_{i m} R_{j n} R_{k p} R_{l q} M_{m n p q}^{0}$, where $\mathbb{H}^{0}$ and $\mathbb{M}^{0}$ are the anisotropic fourth order tensors defined in the frame related to the orthotropic directions of the matrix. The relations between the nonzero components of $\mathbb{H}^{0}$ and those of $\mathbb{M}^{0}$ (directly obtained from (5)) are (using Voigt's notation):

$$
\begin{aligned}
& H_{11}^{0}=-\frac{4}{9 \Delta}\left(-M_{11}^{0}+2 M_{22}^{0}+2 M_{33}^{0}\right) ; \quad H_{22}^{0}=-\frac{4}{9 \Delta}\left(2 M_{11}^{0}-M_{22}^{0}+2 M_{33}^{0}\right) \\
& H_{33}^{0}=-\frac{4}{9 \Delta}\left(2 M_{11}^{0}+2 M_{22}^{0}-M_{33}^{0}\right) ; \quad H_{44}^{0}=\frac{1}{M_{44}^{0}} ; \quad H_{55}=\frac{1}{M_{55}^{0}} ; \quad H_{66}^{0}=\frac{1}{M_{66}^{0}} \\
& \Delta=\left(M_{11}^{0}\right)^{2}+\left(M_{22}^{0}\right)^{2}+\left(M_{33}^{0}\right)^{2}-2 M_{11}^{0} M_{22}^{0}-2 M_{11}^{0} M_{33}^{0}-2 M_{22}^{0} M_{33}^{0}
\end{aligned}
$$

\section{Appendix B. Determination of parameters $\kappa$ and $r$}

Let us consider the determination of $\kappa$ and $r$. The exact point corresponding to $B \rightarrow 0$ is defined by:

$$
\begin{aligned}
& \frac{\Sigma_{h}^{*}}{\sigma_{0}}=\frac{1}{3 x_{2}} \lim _{B \rightarrow 0}\left(\frac{\partial \Pi}{\partial \boldsymbol{D}}\right)=\frac{1}{x_{2}} \frac{1}{|\Omega|} \int_{\Omega} \frac{2}{3 A_{\mathrm{eq}}} \boldsymbol{A}: \mathbb{H}: \boldsymbol{d}^{E} \mathrm{~d} V=\frac{2}{3} \frac{\boldsymbol{A}: \mathbb{H}: \boldsymbol{Q}}{A_{\mathrm{eq}}}\left(\alpha_{2}-\alpha_{1}\right) \\
& \frac{\overline{\boldsymbol{\Sigma}}}{\sigma_{0}}=\lim _{B \rightarrow 0}\left(\frac{\partial \Pi}{\partial \overline{\boldsymbol{D}}}\right)=\frac{2}{3} \frac{\boldsymbol{A}: \mathbb{H}}{A_{\mathrm{eq}}}(1-f)
\end{aligned}
$$

Combining these two relations, it can be shown that:

$$
\Sigma_{h}^{*}=\frac{\alpha_{2}-\alpha_{1}}{1-f} \boldsymbol{\Sigma}: \boldsymbol{Q} ; \quad \Sigma_{\mathrm{eq}}=\sigma_{0}(1-f)
$$

Applying $B \rightarrow 0$ in the approximate solution (24) yields:

$$
\left\{\begin{array}{l}
3 \Sigma_{h}^{*}=\frac{p}{x_{2}} \frac{\partial \Pi}{\partial \widetilde{B}}=p \sigma_{0}\left(\operatorname{arcsinh}\left(u_{2} \chi\right)-\operatorname{arcsinh}\left(u_{1} \chi\right)\right) \\
\overline{\boldsymbol{\Sigma}}-\frac{3 \kappa}{p^{2}} \Sigma_{h}^{*} \mathbb{H}: \boldsymbol{Q}=-\frac{2}{3} \frac{A: \mathbb{H}^{*}}{A_{\mathrm{eq}}} x_{2}\left(\frac{\sqrt{1+u_{2}^{2} \chi^{2}}}{u_{2}}-\frac{\sqrt{1+u_{1}^{2} \chi^{2}}}{u_{1}}\right)
\end{array}\right.
$$

where $\chi=\frac{q}{p} \frac{\boldsymbol{A}: \mathbb{H}: \boldsymbol{Q}}{\widetilde{A}}$. Using the following identity: $\boldsymbol{A}: \mathbb{H}^{*}: \boldsymbol{Q}=\left(1-\frac{9 r h_{2}}{4 p^{2}}\right) \boldsymbol{A}: \mathbb{H}: \boldsymbol{Q}$ and combining relations (B.3) yields:

$$
\left\{\begin{array}{l}
\frac{\boldsymbol{\Sigma}: \boldsymbol{Q}}{\sigma_{0}}-\frac{27 \kappa h_{2}}{4 p^{2}} \frac{\Sigma_{h}^{*}}{\sigma_{0}}-\frac{2 x_{2}^{2}}{3 u_{2} u_{1} p \kappa}\left(p^{2}-\frac{9 r h_{2}}{4}\right) \sinh \left\{\frac{3 \Sigma_{h}^{*}}{p \sigma_{0}}\right\}=0 \\
\frac{\Sigma_{\mathrm{eq}}^{2}}{\sigma_{0}^{2}}+k_{1}\left(\frac{\Sigma_{h}^{*}}{\sigma_{0}}\right)^{2}+k_{2}\left(\frac{\boldsymbol{\Sigma}: \boldsymbol{Q}}{\sigma_{0}}\right)^{2}-2 k_{3} \frac{\Sigma_{h}^{*}}{\sigma_{0}} \frac{\boldsymbol{\Sigma}: \boldsymbol{Q}}{\sigma_{0}}+\frac{2 x_{2}^{2}}{u_{2} u_{1}} \cosh \left(\frac{3 \Sigma_{h}^{*}}{p \sigma_{0}}\right)-\frac{x_{2}^{2}}{u_{2}^{2}}-\frac{x_{1}^{2}}{u_{1}^{2}}=0
\end{array}\right.
$$

Since $\Sigma_{h}^{*}$ is assumed to be low, we propose to develop the hyperbolic terms in (B.4) at the second order. The identification of $\kappa$ and $r$ is made by replacing in (B.4), $\Sigma_{h}^{*}$ and $\Sigma_{\text {eq }}$ by their exact expressions given by (B.2). The two equations (B.4) then become:

$$
\left\{\begin{array}{l}
\frac{1-f}{\alpha_{2}-\alpha_{1}}-\frac{2 x_{2}^{2}}{u_{2} u_{1} \kappa}-\frac{9 h_{2}}{2 \kappa p^{2}}\left\{\frac{3 \kappa^{2}}{2}-\frac{r x_{2}^{2}}{u_{2} u_{1}}\right\}=0 \\
\frac{r(1-f)^{2}}{6\left(\alpha_{2}-\alpha_{1}\right)^{2}}-\kappa \frac{1-f}{\alpha_{2}-\alpha_{1}}+\frac{x_{2}^{2}}{u_{2} u_{1}}+\frac{9 h_{2}}{4 p^{2}}\left\{\frac{3 \kappa^{2}}{2}-\frac{r x_{2}^{2}}{u_{2} u_{1}}\right\}=0
\end{array}\right.
$$


Since (B.5) must be verified whatever the value of $h_{2}$, four equations can be deduced from (B.5) and have to fulfilled by $\kappa$ and $r$. The solution reads:

$$
\kappa=\frac{2 x_{2}^{2}\left(\alpha_{2}-\alpha_{1}\right)}{u_{2} u_{1}(1-f)} ; \quad r=\frac{6 x_{2}^{2}\left(\alpha_{2}-\alpha_{1}\right)^{2}}{u_{2} u_{1}(1-f)^{2}}
$$

\section{References}

[1] A.L. Gurson, Continuum theory of ductile rupture by void nucleation and growth: Part I-Yield criterion and flow rules for porous ductile media, J. Engrg. Mater. Tech. 99 (1977) 2-15.

[2] M. Gologanu, J.-B. Leblond, J. Devaux, Approximate models for ductile metals containing non-spherical voids—case of axisymmetric prolate ellipsoidal cavities, J. Mech. Phys. Solids 41 (11) (1993) 1723-1754.

[3] M. Gologanu, J.-B. Leblond, J. Devaux, Approximate models for ductile metals containing non-spherical voids-case of axisymmetric oblate ellipsoidal cavities, J. Engrg. Mater. Tech. 116 (1994) 290-297.

[4] M. Gologanu, J.-B. Leblond, G. Perrin, J. Devaux, Recent extensions of Gurson's model for porous ductile metals, in: P. Suquet (Ed.), Continuum Micromechanics, Springer-Verlag, Berlin/New York, 1997, pp. 61-130.

[5] M. Garajeu, J.C. Michel, P. Suquet, A micromechanical approach of damage in viscoplastic materials by evolution in size, shape and distribution of voids, Comput. Methods Appl. Mech. Engrg. 183 (2000) 223-246.

[6] A. Benzerga, J. Besson, Plastic potentials for anisotropic porous solids, Eur. J. Mech. A/Solids 20 (2001) 397-434.

[7] K. Liao, J. Pan, S. Tang, Approximate yield criteria for anisotropic porous ductile sheet metals, Mech. Mater. 26 (1997) $213-226$.

[8] D. Wang, J. Pan, An anisotropic Gurson yield criterion for porous ductile sheet metals with planar anisotropy, Internat. J. Damage Mech. 13 (2004).

[9] R. Hill, A theory of yielding and plastic flow of anisotropic solids, Proc. Roy. Soc. London A 193 (1948) $281-297$.

[10] H.S. Son, Y.S. Kim, Prediction of forming limits for anisotropic sheets containing prolate ellipsoidal voids, Internat. J. Mech. Sci. 45 (2003) $1625-1643$. 